\title{
Progression of Corneal Endothelial Insult by Chronic Elevation of intraocular Pressure: A prospective Observational Comparative Study Among Primary Open Angle Glaucoma Patients
}

Elshimaa A.Mateen ( $\nabla$ elshimaa.moussa@yahoo.com )

Sohag University Faculty of Medicine

Hatem Gamal Ammar

Sohag University Faculty of Medicine

Khulood Muhammad Sayed

Sohag University Faculty of Medicine

\section{Research Article}

Keywords: IOP, POAG, Specular microscopy, VF, OCT disc

Posted Date: May 27th, 2021

DOI: https://doi.org/10.21203/rs.3.rs-190994/v1

License: (c) (i) This work is licensed under a Creative Commons Attribution 4.0 International License.

Read Full License 


\section{Abstract}

Purpose: To evaluate specular microscopic changes of corneal endothelial cells (CEC) count and morphology in correlation to retinal nerve fiber layer RNFL changes detected by visual field (VF) and OCT in early and advanced primary open-angle glaucoma (POAG).

Methods: A prospective observational comparative study was conducted on patients with POAG versus non-glaucomatous patients of the same age group. Specular microscopy, VF test, OCT scans of RNFL, and macular ganglion cell complex (GCC) were performed. Glaucoma group was further subdivided into early and advanced stages.

Results: The study included 130 eyes of 130 subjects, 70 were eyes with POAG (glaucoma group), 60 were healthy eyes (control group). Both groups were comparable regarding mean age and sex.

In the early glaucoma group, a significant negative correlation was found between the coefficient of variation $(\mathrm{CV} \%)$ and superior PRNFL thickness $(r=-0.5, p$-value $=0.018)$. A significant negative correlation was also found between percentage of cellular hexagonality (HEX\%) and vertical cup/disc (C/D) ratio $(r=-0.43$, p-value $=0.035)$. A significant positive correlation was found between HEX\% and (superior, inferior) PRNFL thickness $(r=0.53,0.5$ and $p$-value $=0.008,0.015)$ respectively.

Mean CEC count was significantly lower in the advanced glaucoma group than in the control group $(2958.7 \pm 371.2$ vs $3085 \pm 172.5$, p value $=0.043)$.

Conclusions: $C E C$ is affected by chronic intraocular pressure (IOP) elevation just like the PRNFL and macular GCC. CEC morphology is insulted in the early stages of POAG while the count can withstand chronic IOP elevation till advanced stages were both are affected.

\section{Introduction}

One of the unique characters of the cornea is being clear and transparent, which is dependent on its stromal anatomical structure and the function of the most inner hexagonal endothelial cell layer ${ }^{1,2}$. Corneal endothelial cell (CEC) loss is irreversible and to maintain corneal transparency, preservation of these cells is a must ${ }^{3}$. Variable causes can insult corneal endothelium resulting in corneal decompensation and opacification like trauma, cataract surgery, and diseases such as diabetes and glaucoma ${ }^{4-6}$.

Glaucoma is a potentially blinding disease of variable types, primary or secondary which is associated with optic neuropathy and retinopathy in addition to its effect on corneal endothelial cell ${ }^{7,8}$. Endothelial cell loss in glaucoma can be attributed to the direct effect of compressing elevated intraocular pressure (IOP), the toxicity of medications, and the effect of surgeries and LASER procedures used to control IOP'11. 
Glaucoma and cataract are more prevalent and commonly coexist among the elderly population, in whom CEC evaluation is mandatory to check its ability to withstand the surgical trauma of cataract surgeries, in which endothelial cell loss is an inevitable consequence ${ }^{12}, 13$.

The effect of acute IOP rise on corneal endothelium is documented in previous reports ${ }^{14}$, but little information is available about the effect of chronic elevation of IOP on corneal endothelial cells (count and morphology) in primary open angle glaucoma (POAG). In our study, we aimed to study CEC affection in early and advanced POAG and to find out if there are any correlations between changes occurring in the retinal nerve fiber layer (RNFL), macular ganglion cell complex (GCC) on one side, and changes of corneal endothelial cell (count and morphology) on the other side in glaucoma patients. To the best of our knowledge, such a study has not been done before.

\section{Patient And Method}

\section{Study Design}

A prospective observational comparative study was conducted on patients with POAG (controlled on medical treatment) versus non-glaucomatous patients of the same age group.

\section{Patient Selection}

After the approval of the Human Ethical Committee in Sohag faculty of Medicine; written consent was taken from all patients who participated in this study after explanation of the procedure and nature of the disease. The tenets of the Helsinki Declaration were followed.

The study included 130 eyes of 130 subjects, 70 were eyes with POAG (glaucoma group) , 60 were healthy eyes (control group). The right eye was included in the control and glaucoma groups. In the glaucoma group, IOP was controlled for at least 6 months with one or more of the following antiglaucoma medications (Prostaglandin analogs, Beta-blockers, Alpha agonists, Carbonic anhydrase inhibitor eye drops). The study population attended to Sohag ophthalmic investigation center for their routine scheduled follow up (VF, OCT PRNFL, and macular GCC) in the period from June 2019 to December 2019.

Glaucoma Staging System 2 (GSS 2) was used for the classification of glaucoma, it is based on two main perimetric global indices Mean Deviation [MD] and Pattern Standard Deviation [PSD], plotted on an $X-Y$ coordinate. It classifies visual field defects in 6 stages (stage 0 which includes normal population, a borderline stage which is a stage defined between stage 0 and one, and 5 consecutive stages from (1:5). according to damage severity. Classification also includes 3 types of defects (generalized, localized, and mixed). ${ }^{15}$

In this study, we considered stages one and two GSS2 as an early stage (40 eyes) and groups three, four, and five GSS2 an advanced group of glaucoma (30 eyes). 
Sixty control subjects were the relatives of the patients and workers in Sohag ophthalmic investigations center of the same age group. The control group was examined two times for IOP measurement in two different occasions. They were also tested for VF, RNFL thickness to exclude normal tension glaucoma.

\section{Inclusion Criteria:}

Eyes with medically controlled IOP measurement for at least six months $(15.5 \mathrm{~mm} \mathrm{Hg}+/-2.6 \mathrm{~mm} \mathrm{Hg})$ by Goldman applanation tonometry ${ }^{16}$. Eyes with cup/disc ratio (C/D) ratio $\geq 0.7$, or asymmetry $>0.2$, or difference in Vertical (C/D) and Horizontal (C/D) >0.2. Notching, or disc hemorrhage, or excavation reaching disc margin. Glaucomatous visual field (VF) changes (stages 1:5 according to GSS2). Peripapillary RNFL thinning. Visual acuity (VA) of 0.4 Log MAR or more were included.

\section{Exclusion Criteria:}

Eyes with juvenile open-angle glaucoma, angle-closure glaucoma, normal-tension glaucoma, pseudo exfoliation syndrome (PEX). chronic contact lens wearers, ametropia $>+/-6 D$, myopic fundus changes, media opacities, any neurological disorders, previous operations or retinal pathologies, unreliable VF tests, and OCT images of signal strength less than $60{ }^{17}$.

All eyes were subjected to VF examination using standard automated perimetry (SAP) Humphrey Field Analyzer (HFA) II 750 (Carl Zeiss Meditec Inc., Dublin, California) 30-2 test with Standard Swedish Interactive Thresholding Algorithm (SITA) Strategy. SAP tests were classified following Anderson and Patella criteria.

OCT of the optic disc (ONH/RNFL scan) and macular ganglion cell layer (GCC, ganglion cell complex segmentation) was performed using RTVue (Optovue Inc., Fermont, CA).

Specular microscopy (SP-2000P; TOPCON, Tokyo, Japan). Images were captured from the central cornea (central measurement) and analyzed using IMAGEnet 2000 software (TOPCON). Endothelial (CED), (HEX\%), and (CV\%) were determined. All examinations were performed by the same examiner who was masked to the participant's condition. Multiple images are taken by a central view for each eye to obtain a clear image of a good quality of the central endothelium, in which the centers of at least 100 contiguous endothelial cells were marked using software available in the system with clear cell boundaries.

\section{Statistics:}

Software version (26.0) (SPSS, Inc., Chicago, IL, USA) was used. All participants were chosen by using the systematic random sample technique from the attendees who fulfilled the inclusion criteria.

The data were tested for normality using the Kolmogorov-Smirnov test and for homogeneity variances before further statistical analysis. 
Differences between groups for continuous measures were analyzed by an independent sample t-test and the chi-squared test for categorical measures. $Z$ test was used to compare 2 proportions.

Pearson's correlation coefficients $(r)$ were used to assess the correlations between variables of corneal parameters, VF, and OCT disc parameters. A p-value $\leq 0.05$ was considered statistically significant.

\section{Results}

The study included 130 eyes of 130 subjects, 70 were eyes with POAG (40 eyes were early glaucoma group and 30 eyes were advanced glaucoma group), 60 were healthy eyes (control group), who were the relatives of the patients and workers in Sohag ophthalmic investigations center of the same age group.

Both groups were comparable regarding mean age ( $58.5 \pm 9.42$ vs $59.5 \pm 8.4$ years, $p$-value $=0.5)$ and sex (males were $30.8 \%$ vs $26.66 \%$, $p$-value $=0.6$ ) for glaucoma and control groups, respectively.

Different parameters of specular microscopy, OCT disc, and VF are summarized in (tables 1,2).

Table (1): Corneal parameters assessed by specular microscopy,

\begin{tabular}{|lllll|}
\hline & $\begin{array}{l}\text { Control group } \\
(46.15 \%)\end{array}$ & $\begin{array}{l}\text { Early stage } \\
(\text { Stage 1 \& } 2)\end{array}$ & $\begin{array}{l}\text { Advanced stage } \\
(\text { Stage 3, 4 \& 5) }\end{array}$ & p-value \\
& & $(36.92 \%)$ & $(17 \%)$ & \\
\hline Central corneal thickness (CCT) & $518.53 \pm 44.14$ & $538.4 \pm 40.6$ & $515.33 \pm 16.9$ & 0.174 \\
\hline Cell density (CED) & $3085 \pm 172.56$ & $2943.9 \pm 449.8$ & $2958.73 \pm 371.2$ & 0.569 \\
\hline Coefficient of variation (CV) & $40.5 \pm 20.8$ & $35.52 \pm 4.30$ & $38.20 \pm 7.54$ & 0.454 \\
\hline Hexagonality (HEX) & $43.1 \pm 14.6$ & $45.04 \pm 9.15$ & $40.90 \pm 7.93$ & 0.622 \\
\hline Vertical (C:D) ratio & $0.46 \pm 0.26$ & $0.56 \pm 0.65$ & $0.69 \pm 0.31$ & 0.001 \\
\hline Cup volume & $0.20 \pm 0.28$ & $0.34 \pm 0.232$ & $0.27 \pm 0.267$ & 0.149 \\
\hline
\end{tabular}

Data represented by (\%) percentage, CED; corneal endothelial cell density, (HEX\%); percentage of hexagonality, (CV\%); co-efficient of variation of the cell area, (mean \pm SD); p-value calculated using ANOVA.

Table (2): OCT disc and visual field parameters, 


\begin{tabular}{|c|c|c|c|c|}
\hline & $\begin{array}{l}\text { Control group } \\
(46.15 \%)\end{array}$ & $\begin{array}{l}\text { Early stage } \\
\text { (Stage } 1 \text { \& 2) } \\
(36.92 \%)\end{array}$ & $\begin{array}{l}\text { Advanced } \\
\text { stage } \\
\text { (Stage 3, } 4 \text { \& } \\
5) \\
(17 \%)\end{array}$ & $\begin{array}{l}\mathrm{p} \text { - } \\
\text { value }\end{array}$ \\
\hline Superior RNFL thickness & $\begin{array}{l}119.06 \\
\pm 26.64\end{array}$ & $\begin{array}{l}110.064 \\
\pm 31.22\end{array}$ & $61.09 \pm 24.014$ & 0.003 \\
\hline Inferior RNFL thickness & $\begin{array}{l}124.064 \\
\pm 20.19\end{array}$ & $\begin{array}{l}112.52 \\
\pm 36.42\end{array}$ & $60.36 \pm 38.47$ & 0.003 \\
\hline Temporal RNFL thickness & $75.22 \pm 12.13$ & $66.98 \pm 18.42$ & $70.09 \pm 36.04$ & 0.042 \\
\hline Nasal RNFL thickness & $76.42 \pm 10.46$ & $\begin{array}{l}74.34 \\
\pm 21.037\end{array}$ & $71.32 \pm 44.27$ & 0.033 \\
\hline Average macular GCC thickness & $96.64 \pm 10.47$ & $92 \pm 13.34$ & $67.54 \pm 24.73$ & 0.000 \\
\hline Superior macular GCC thickness & $95.42 \pm 9.33$ & $90.58 \pm 13.64$ & $70 \pm 22.54$ & 0.000 \\
\hline Inferior macular GCC thickness & $97.74 \pm 13.14$ & $93.29 \pm 13.60$ & $65.18 \pm 26.97$ & 0.000 \\
\hline Mean deviation (MD) of the $V / F$ & $-1.63 \pm 3.14$ & $-4.21 \pm 1.28$ & $-19.89 \pm 9.86$ & 0.000 \\
\hline $\begin{array}{l}\text { Pattern standard deviation (PSD) of the } \\
\text { V/F }\end{array}$ & $2.10 \pm 2.33$ & $3.68 \pm 1.43$ & $8.38 \pm 3.20$ & 0.000 \\
\hline
\end{tabular}

Data represented by (\%) percentage, RNFL; Retinal nerve fiber layer, GCC; Ganglion cell complex, V/F; Visual field, (C:D); Cup to disc ratio, (mean \pm SD); p-value calculated using ANOVA.

No significant difference was found between corneal parameters in control versus glaucoma groups, except for the CEC density which was significantly lower in the advanced glaucoma group $(2958.7 \pm 371.2)$ than that of the control group $(3085 \pm 172.5)(P$ value $=0.043)$

In the early glaucoma group, a significant negative correlation was found between (CV\%) and superior RNFL thickness $(r=-0.5, p$-value $=0.018)$. A significant negative correlation was also found between $(\mathrm{HEX} \%)$ and vertical (C/D) ratio ( $r=-0.43$, $p$-value $=0.035)$.

Positive correlations were found between HEX\% and (superior, inferior) PRNFL thickness in early glaucoma $(r=0.53,0.5$ and $p$-value $=0.008,0.015)$ respectively. (table 3 and figures 1,2 and 3 ).

Table (3): Correlations between different parameters in early glaucoma group, 


\begin{tabular}{|llll|}
\hline & & R & P value \\
\hline CV \% & Superior RNFL thickness & -0.5 & $0.018^{\star}$ \\
\hline HEX\% & Vertical (C/D) ratio & -0.43 & $0.035^{\star}$ \\
\hline HEX\% & Superior RNFL thickness & 0.53 & $0.008^{\star}$ \\
\hline HEX\% & Inferior RNFL thickness & 0.5 & $0.015^{\star}$ \\
\hline
\end{tabular}

$\mathrm{CV} \%$; Percentage of coefficient of variation of corneal endothelial cell, HEX\%; Percentage of hexagonality of corneal endothelial cells, RNFL; Retinal nerve fiber layer, $(R)$ correlation, *P. value $<0.05$ statistically significant.

In the advanced glaucoma group; correlations couldn't be found between RNFL changes and corneal endothelial cell changes.

\section{Discussion}

The study aims to evaluate and compare corneal endothelial cell changes (count and morphology) in response to chronic IOP elevation in early vs advanced POAG. We also aimed to assess the correlation between corneal endothelial cell changes and RNFL changes as detected by VF and OCT.

To the best of our knowledge, such a study had not been done before. Previous studies discussed corneal endothelial changes in correlation to acute IOP elevation in ACG, the use of anti-glaucoma medications, and anti-glaucoma surgical procedures ${ }^{18-20}$.

Both groups (Control and glaucoma groups) were comparable regarding mean age ( $58.5 \pm 9.42$ vs 59.5 \pm 8.4 years, $p$-value $=0.5$ ) to exclude the age as confounding factor, since POAG is usually seen at older age asscociated with simultaneous age-related endothelial cell loss.

The study revealed a significant negative correlation between CV\% and superior PRNFL thickness and also between HEX\% and vertical C/D, which means that the progressive thinning of the superior PRNFL which is 1 stly affected by glaucoma ${ }^{21}$, and progressive increase in vertical (C/D), both are accompanied by progressive deterioration of corneal morphology while endothelial CEC density remains unaffected. Corneal endothelial cells morphology changed in the form of an elevated or abnormal rate of polymegathism and decreased the number of functioning hexagonal endothelial cells as a result of the physiological stress to the corneal endothelium, these findings were different from that found by Sushma Verma et al, who stated morphological changes like CV\% and HEX\% were not significantly different across the angle-closure disease spectrum ${ }^{3}$. This discrepancy in results may be due to the different nature of both types of glaucoma. Angle-closure glaucoma (ACG) is characterized by acute attacks in which severe and sudden closure of the angle with very high IOP, decreased visual acuity and corneal edema with significant endothelial cell loss ${ }^{22}$. 
This study revealed early affection of $\mathrm{CV} \%$ and $\mathrm{HEX} \%$ but not CEC density, which occurred in the interval period of chronic IOP elevation before the diagnosis of glaucoma has been made. Yu ZY et al reported that elevated IOP cause destruction of the HEX\% and the damage of the cells causes further deformation and loss, but he didn't correlate these changes of corneal endothelial cells to PRNFL thickness nor macular GCC thickness 23

Morphological changes detected in early glaucoma with controlled IOP could be attributed only to IOP changes as eyes with previous surgical and LASER procedures-known to affect corneal endothelium in other studies were excluded ${ }^{24-26}$. The effect of topical antiglaucoma medication on corneal endothelium is negligible according to Kwou-Yeung Wu et al, ${ }^{19}$.

The study revealed a significant reduction of CEC density in advanced glaucoma when compared to the control group. The significantly lower CEC density in the advanced glaucoma group is consistent with the literature proved low CEC density among patients with glaucoma, ocular hypertensive glaucoma, and normal-tension glaucoma. Early reduction of CEC density in patients with ACG was reported by Sushma Verma et al which is attributed to the different nature and course of $\mathrm{ACG}^{3}, 17,27$.

In the advanced glaucoma group; correlations couldn't be found between RNFL changes and corneal endothelial cell changes, it could be due to the floor effect of marked thinning of the RNFL to a certain level that makes the correlations difficult to appear ${ }^{28,29}$.

From this study, we can conclude that corneal endothelial cells are insulted in POAG with corneal cell morphology affected in early stages of glaucoma while the cell count can withstand chronic IOP elevation till advanced stages were both morphology and count are affected.

This conclusion could be helpful in surgical concerns, especially that most glaucomatous patients would need cataract surgery in different periods of their lives ${ }^{12,30}$.

Patients with cataract and early controlled POAG in whom specular microscopy is difficult to be done as in bedridden and intellectual disability paients, patients with neurological diseases associated with head nodding and severe blepharospasm) cataract surgeries could be done safely relying on the fact that (CEC) density remains within the normal range in early controlled POAG.

However, there were some limitations in the study as it did not include another group of ACG to compare and correlate to control and POAG groups.

Further studies should be done including a larger number of eyes and different types of glaucoma to understand the effect of different types of glaucoma on corneal endothelial cells.

\section{Declarations}


There is no conflict of interest. There is neither a financial relationship nor sponsorship with any organization to be declared. The authors have full control of all primary data and they agree to allow the Journal to review their data if required.

\section{References}

1. Feizi S. Corneal endothelial cell dysfunction: etiologies and management. Ther Adv Ophthalmol 2018; 10: $2515841418815802-2515841418815802$. DOI: 10.1177/2515841418815802.

2. Sridhar MS. Anatomy of cornea and ocular surface. Indian J Ophthalmol 2018; 66: 190-194. DOI: 10.4103/ijo.IJO_646_17.

3. Verma S, Nongpiur ME, Husain R, et al. Characteristics of the Corneal Endothelium Across the Primary Angle Closure Disease Spectrum. IOVS 2018; 59: 4525-4530. DOI: 10.1167/iovs.18-24939.

4. Aldrich BT, Schloetzer-Schrehardt U, Skeie JM, et al. Mitochondrial and morphologic alterations in native human corneal endothelial cells associated with diabetes mellitus. IOVS 2017; 58: 2130-2138.

5. Ventura ACS, Wälti R and Böhnke M. Corneal thickness and endothelial density before and after cataract surgery. BJO 2001; 85: 18. DOI: 10.1136/bjo.85.1.18.

6. Wang PX, Koh VT and Loon SCJAo. Laser iridotomy and the corneal endothelium: a systemic review. 2014; 92: 604-616.

7. SETÄLÄ K. Corneal endothelial cell density after an attack of acute glaucoma. Acta Ophthalmol 1979; 57: 1004-1013.

8. Gagnon M-M, Boisjoly HM, Brunette I, et al. Corneal endothelial cell density in glaucoma. Cornea 1997; 16: 314-318.

9. Pastor SA, Williams R, HETHERINGTON J, et al. Corneal endothelial cell loss following trabeculectomy with mitomycin C. J Glaucoma 1993; 2: 112-113.

10. Chen M-J, Liu CJ-L, Cheng C-Y, et al. Corneal status in primary angle-closure glaucoma with a history of acute attack. J Glaucoma 2012; 21: 12-16.

11. Baratz KH, Nau CB, Winter EJ, et al. Effects of glaucoma medications on corneal endothelium, keratocytes, and subbasal nerves among participants in the ocular hypertension treatment study. Cornea 2006; 25: 1046-1052.

12. Osman AA, El-Sayed SM and Fahmy AE. Corneal Endothelial Changes by Specular Microscopy after Uncomplicated Phaco-Emulsification of Hard Senile Cataract Graded by Pentacam. EJHM 2019; 76: 4474-4482.

13. Ling JD and Bell NP. Role of Cataract Surgery in the Management of Glaucoma. Int Ophthalmol Clin 2018; 58: 87-100. DOI: 10.1097/II0.0000000000000234.

14. Higa A, Sakai H, Sawaguchi S, et al. Corneal endothelial cell density and associated factors in a population-based study in Japan: the Kumejima study. AJO 2010; 149: 794-799.

15. Brusini P and Filacorda S. Enhanced Glaucoma Staging System (GSS 2) for classifying functional damage in glaucoma. J Glaucoma 2006; 15: 40-46. 
16. Hashemi $\mathrm{H}$, Kashi A, Fotouhi $A$, et al. Distribution of intraocular pressure in healthy Iranian individuals: the Tehran Eye Study. BJO 2005; 89: 652-657.

17. Tan NY, Tham Y-C, Koh V, et al. The effect of testing reliability on visual field sensitivity in normal eyes: the Singapore Chinese Eye Study. Ophthalmology 2018; 125: 15-21.

18. Wagdy FM, Zaky AG and Gohar SN. Corneal endothelial changes after subscleral trabeculectomy with mitomycin-C. DJO 2019; 20: 63.

19. Wu K-Y, Wang H-Z and Hong S-J. Cellular cytotoxicity of antiglaucoma drugs in cultured corneal endothelial cells. KJMS 2007; 23: 105-111.

20. Janson BJ, Alward WL, Kwon YH, et al. Glaucoma-associated corneal endothelial cell damage: A review. Surv Ophthalmol 2018; 63: 500-506.

21. Lee JW, Yau GS, Woo TT, et al. The association between macular thickness and peripapillary retinal nerve fiber layer thickness in Chinese children. Medicine 2015; 94.

22. Khaw PT, Shah P and Elkington AR. Glaucoma-1: diagnosis. BMJ 2004; 328: 97-99. DOI: 10.1136/bmj.328.7431.97.

23. Yu Z-Y, Wu $L$ and Qu B. Changes in corneal endothelial cell density in patients with primary openangle glaucoma. World J Clin Cases 2019; 7: 1978-1985. DOI: 10.12998/wjcc.v7.i15.1978.

24. El Rehim DAEF, El-Hessy A-A, El-Saeed El-Kholy S, et al. Effect of Ex-PRESS glaucoma filtration device on corneal endothelium in primary open-angle glaucoma. JEOS 2019; 112: 9-13. Original Article. DOI: 10.4103/ejos.ejos_69_18.

25. Toyokawa N, Araki-Sasaki K, Nambu H, et al. Changes of corneal endothelial cell density after EXPRESS glaucoma shunt implantation: 2-year follow-up study. J Clin Ophthalmol 2018; 2: 41.

26. Hong C, Kitazawa $Y$ and Tanishima T. Influence of argon laser treatment of glaucoma on corneal endothelium. Japanese journal of ophthalmology 1983; 27: 567-574. 1983/01/01.

27. Boisjoly H, Mortazavi A, Brunette I, et al. Corneal endothelial cell anomalies in glaucoma suspects with ocular hypertension. Investigative Ophthalmology \& Visual Science 1996; 37: S818.

28. Zhang X, Dastiridou A, Francis BA, et al. Comparison of Glaucoma Progression Detection by Optical Coherence Tomography and Visual Field. AJO 2017; 184: 63-74. 09/28. DOI: 10.1016/j.ajo.2017.09.020.

29. Bowd C, Zangwill LM, Weinreb RN, et al. Estimating Optical Coherence Tomography Structural Measurement Floors to Improve Detection of Progression in Advanced Glaucoma. AJO 2017; 175: 37-44. 11/30. DOI: 10.1016/j.ajo.2016.11.010.

30. Hwang HB, Lyu B, Yim HB, et al. Endothelial Cell Loss after Phacoemulsification according to Different Anterior Chamber Depths. J Ophthalmol 2015; 2015: 210716. DOI: 10.1155/2015/210716.

\section{Figures}




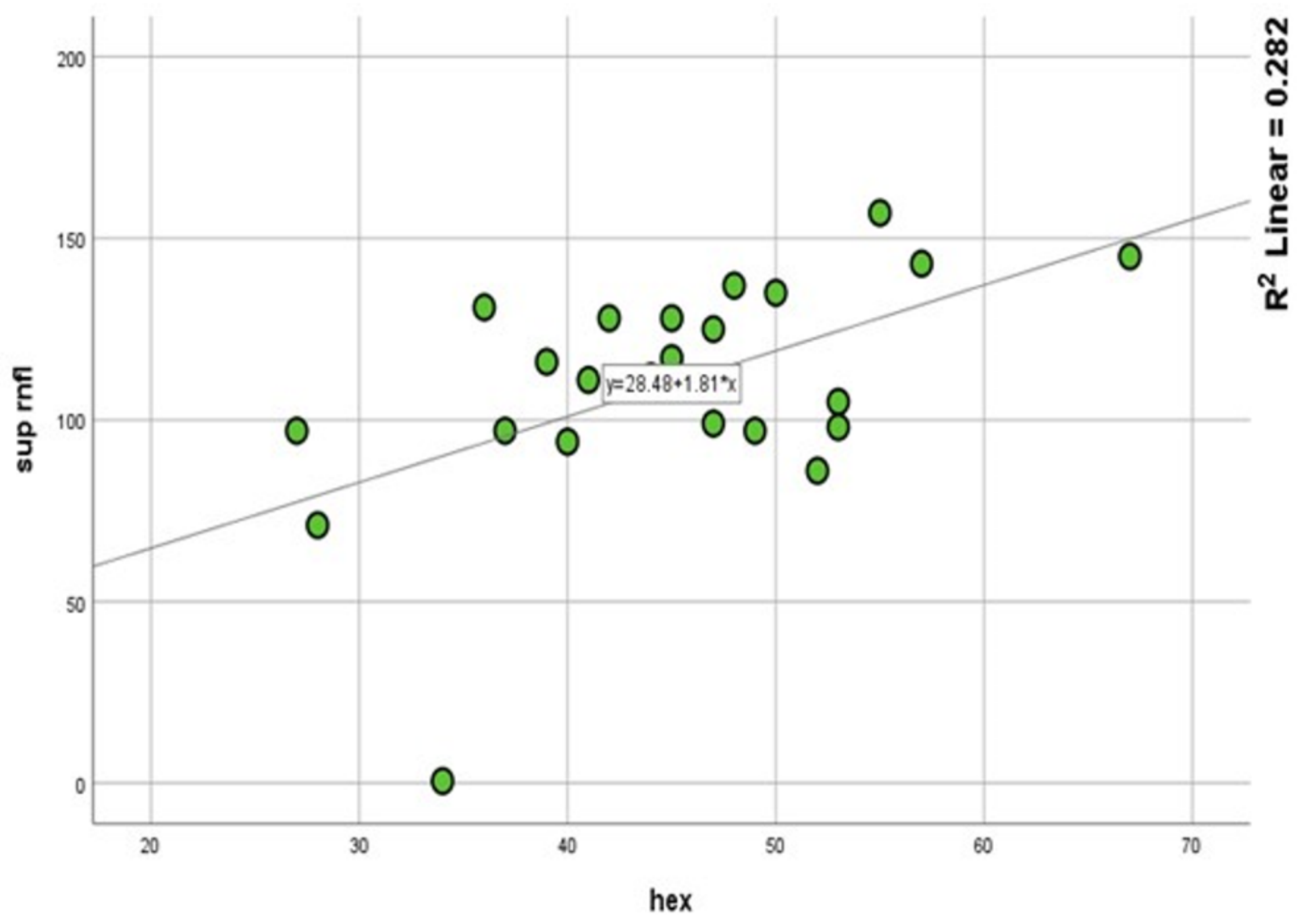

Figure 1

Correlation between (HEX\%) percentage of hexagonality and superior (RNFL) peripapillary retinal nerve fiber layer. 


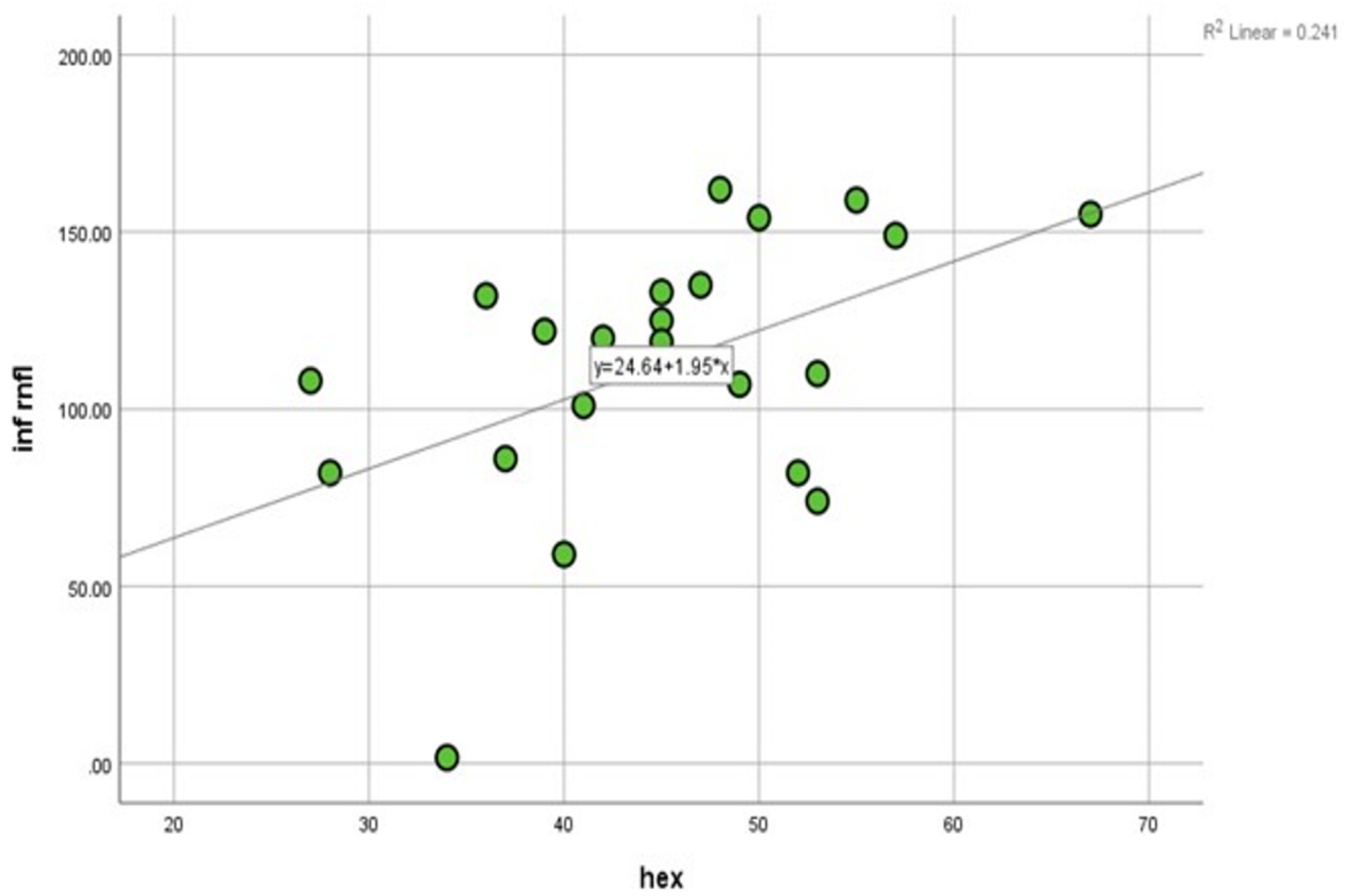

Figure 2

Correlation between (HEX\%) percentage of hexagonality and inferior (RNFL) peripapillary retinal nerve fiber layer. 


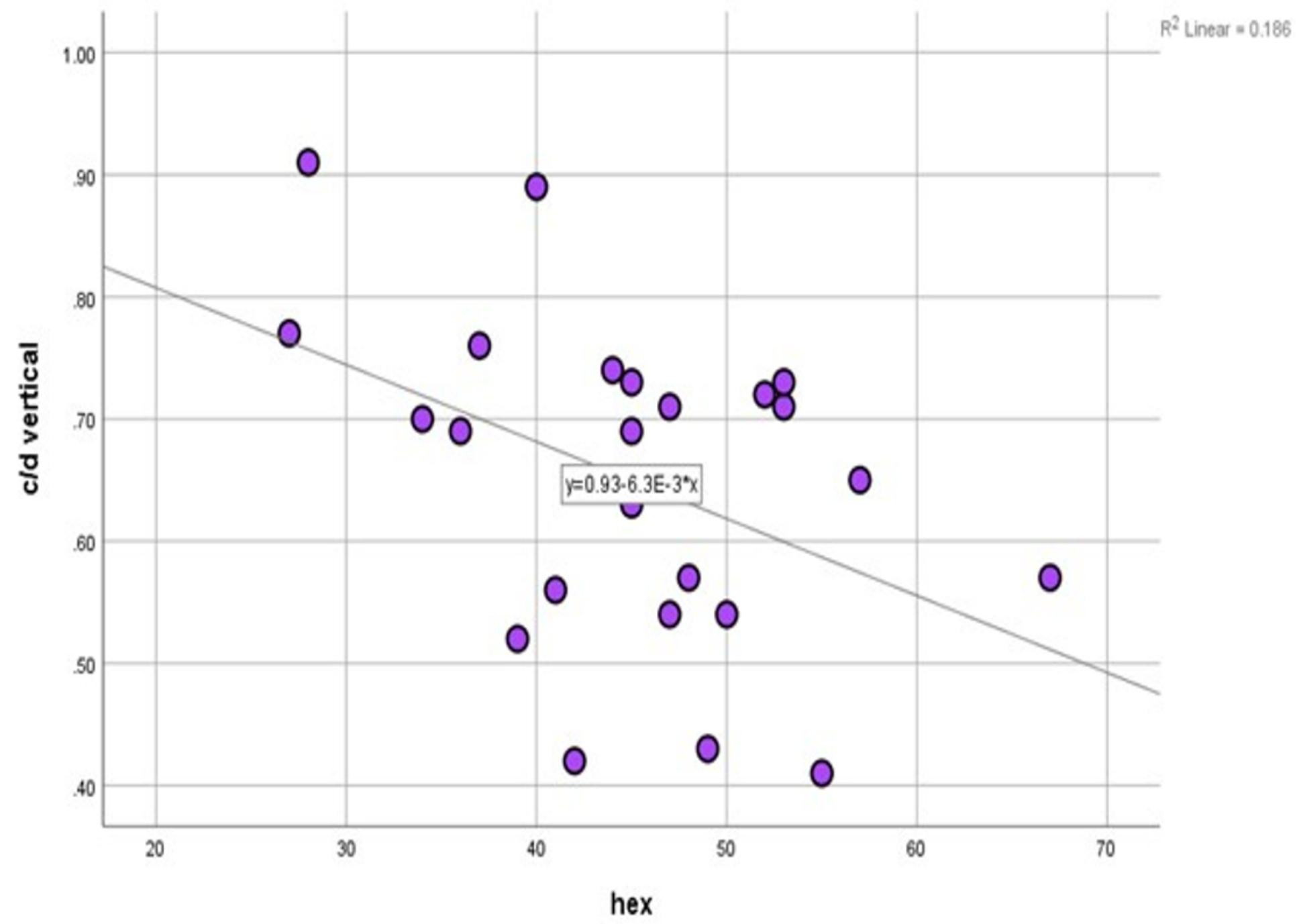

Figure 3

Correlation between (HEX\%) percentage of hexagonality and vertical (C/D) cup to disc ratio. 